\title{
Seletividade de Formulações de Glyphosate Aplicado nos ESTÁDIOS IMATUROS DE Trichogramma pretiosum ${ }^{1}$
}

\author{
Selectivity of Glyphosate Formulations Applied on Immature Stages of Trichogramma Pretiosum
}

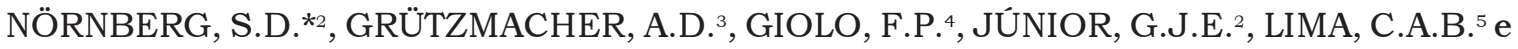 \\ GRÜTZMACHER, D.D. ${ }^{6}$
}

\begin{abstract}
RESUMO - A seletividade de oito formulações comerciais de glyphosate registradas no Brasil (Roundup $^{\circledR}$, Roundup ${ }^{\circledR}$ WG, Roundup ${ }^{\circledR}$ Transorb, Polaris $^{\circledR}$, Gliz ${ }^{\circledR} 480$ CS, Glifosato Nortox ${ }^{\circledR}$, Glifosato 480 Agripec $^{\circledR}$ e Zapp ${ }^{\circledR}$ Qi) foi avaliada sobre os estádios imaturos do parasitóide de ovos Trichogramma pretiosum, bem como seus efeitos sobre os adultos emergidos, em condições de laboratório (temperatura de $25 \pm 1{ }^{\circ} \mathrm{C}$, umidade relativa de $70 \pm 10 \%$, fotofase de $14 \mathrm{~h}$ e luminosidade de $500 \mathrm{lux}$ ). Os bioensaios consistiram na pulverização da calda herbicida, na concentração de $14,4 \mathrm{mg} \mathrm{L}^{-1}$ de equivalente ácido de glyphosate, sobre ovos de Anagasta kuehniella contendo em seu interior o parasitóide nos estádios imaturos de ovolarva, pré-pupa e pupa. Reduções na emergência de adultos em relação à testemunha foram utilizadas para mensurar os efeitos dos tratamentos. Aos adultos do parasitóide emergidos de pupas pulverizadas foram ofertados ovos do hospedeiro A. kuehniella, sendo avaliado o parasitismo de T. pretiosum de cada tratamento em relação à testemunha. Não houve efeito deletério significativo para nenhum dos herbicidas avaliados quando pulverizados sobre os diferentes estádios imaturos de desenvolvimento de T. pretiosum, de acordo com a IOBC. De forma similar, o parasitismo de adultos emergidos de ovos pulverizados na fase de pupa também não foi afetado.
\end{abstract}

Palavras-chave: controle biológico, herbicida, inimigo natural, parasitóide de ovos, toxicidade.

ABSTRACT - The selectivity of eight commercial herbicide products with glyphosate formulations (Roundup ${ }^{\circledR}$, Roundup ${ }^{\circledR}$ WG, Roundup ${ }^{\circledR}$ Transorb, Polaris ${ }^{\circledR}$, Gliz ${ }^{\circledR} 480$ CS, Glifosato Nortox ${ }^{\circledR}$, Glifosato 480 Agripec ${ }^{\circledR}$ and Zapp ${ }^{\circledR}$ Qi) registered in Brazil, was assessed in relation to the immature stages of the egg-parasitoid Trichogramma pretiosum, as well as to their effects on the emerged adults, under laboratory conditions (temperature $25 \pm 1{ }^{\circ} \mathrm{C}$, relative humidity $70 \pm 10 \%$; photophase 14 hours and brightness 500 lux). The bioassays consisted of herbicide spray at the concentration of $14.4 \mathrm{mg} \mathrm{L}^{-1}$ of equivalent gliphosate acid on eggs of Anagasta kuehniella containing the immature stage egg-larva, pre-pupa and pupa of T. pretiosum. Reductions observed in adult emergence, compared to the control, were used to measure the effects of the treatments. Eggs from the host A. kuehniella were presented to the adults of the parasitoids emerged from the sprayed pupae, with the parasitism of $T$. pretiosum being evaluated in each treatment compared to the control. No significant harmful effect was observed for any of the herbicides assessed when sprayed at the different immature stages of development of T. pretiosum, in agreement with IOBC. The parasitism of the adults emerged from eggs sprayed at the pupa stage was not affected either.

Keywords: biological control, herbicide, natural enemy, egg-parasitoid, toxicity.

1 Recebido para publicação em 17.10.2007 e na forma revisada em 18.6.2008.

2 Engọ-Agr ${ }^{0}$, Doutorando, Programa de Pós-Graduação em Fitossanidade, Dep. de Fitossanidade da Faculdade de Agronomia Eliseu Maciel - FAEM da Universidade Federal de Pelotas - UFPel, Bolsista CNPq, autor para correspondência, <sandro_ufpel@hotmail.com>; Caixa Postal 354, 96010-900, Capão do Leão-RS, Brasil; ${ }^{3}$ Engo-Agroํㅡ. Dr., Prof. da FAEM/UPel, bolsista CNPq; ${ }^{4}$ Eng-o-Agr ${ }^{0}$, Dr., Fitossanidade, Syngenta Seeds Ltda.; ${ }^{5}$ Bióloga, Doutoranda, bolsista CAPES no Programa de Pós-Graduação em Fitossanidade, FAEM/UFPel; ${ }^{6}$ Eng ${ }^{-0}-$ Agr $^{0}$, Dr., bolsista PRODOC-CAPES, Programa de Pós-Graduação em Fitossanidade, FAEM/UFPel. 


\section{INTRODUÇÃO}

As plantas daninhas podem reduzir a produtividade das culturas e aumentar os custos de produção quando não controladas, sendo a utilização de herbicidas um dos principais métodos empregados pelos produtores para evitar a competição com as culturas nas lavouras.

O uso de herbicidas de ação total, pósemergência, em culturas como a soja surgiu, entre outros fatores, graças à biotecnologia, que, pela introdução de um gene nessa cultura, proporcionou a resistência ao herbicida glyphosate, tornando possível sua utilização na cultura para controle das plantas daninhas (Siqueira et al., 2004).

Dos herbicidas utilizados nas lavouras, destacam-se principalmente aqueles à base de glyphosate, que são usados no controle de plantas daninhas anuais e perenes (Rodrigues \& Almeida, 2005). O comércio de herbicidas formulados à base de glyphosate tem aumentado nos últimos anos (Shaner, 2000); atualmente, são disponibilizadas no mercado diversas formulações que, apesar de apresentarem o mesmo mecanismo de ação, possuem diferentes sais em sua composição, como sal potássico, sal de isopropilamina e sal de amônio, além da diferenciação entre os ingredientes inertes em cada formulação (Rodrigues \& Almeida, 2005).

Embora existam informações na literatura sobre as propriedades físicas, químicas e toxicológicas do glyphosate (Amarante Júnior \& Santos, 2002), há poucas informações a respeito da toxicidade das formulações comerciais desse produto sobre organismos não-alvo. Moléculas herbicidas, apesar de serem produzidas para uso exclusivo no controle de plantas daninhas, podem causar efeitos secundários não desejados (Rizzardi et al., 2003). Com relação à molécula do glyphosate, resultados demonstraram toxicidade para a microbiota do solo (Santos et al., 2005, 2006) e também sobre insetos benéficos, em trabalhos com parasitóides de ovos do gênero Trichogramma (Hassan et al., 1998; Giolo et al., 2005; Manzoni et al., 2007).

O gênero Trichogramma compreende 160 espécies, que ocorrem em diversos agroecossistemas do mundo (Zucchi \& Monteiro, 1997) e são importantes como agentes de controle natural de lepidópteros-praga em uma grande quantidade de culturas (Hassan et al., 1998). A ação desses insetos, parasitando ovos, impede que seus hospedeiros atinjam a fase larval, que é o estádio que provoca os maiores prejuízos às culturas (Botelho, 1997). A preservação desses organismos nos agroecossistemas torna-se importante para auxiliar a manutenção das populações de insetos-praga abaixo do nivel de dano econômico (Carvalho et al., 2001).

Considerando a ampla representatividade do Trichogramma, o grupo de trabalho da "International Organization for Biological and Integrated Control of Noxious Animals and Plants (IOBC), West Palaearctic Regional Section (WPRS) - escolheu este parasitóide como um gênero-padrão para a realização de testes de seletividade (Hassan et al., 1998). Assim, estudos sobre seletividade de pesticidas a inimigos naturais de pragas devem ser realizados com o intuito de gerar informações que possam auxiliar na tomada de decisão em programas de Manejo Integrado de Pragas (MIP) e na manutenção desses organismos nos agroecossistemas, de modo que estes atuem na regulação de populações de insetos-praga.

Objetivou-se com este trabalho avaliar a seletividade, em condições de laboratório, das principais formulações de glyphosate sobre estádios imaturos de desenvolvimento do parasitóide de ovos $T$. pretiosum, bem como avaliar os efeitos no parasitismo de adultos oriundos de ovos pulverizados no estádio de pupa.

\section{MATERIAL E MÉTODOS}

Os bioensaios foram conduzidos nos laboratórios de Controle Biológico e de Pesticidas do Departamento de Fitossanidade da Faculdade de Agronomia Eliseu Maciel, Universidade Federal de Pelotas (DFs/FAEM/UFPel), PelotasRS, seguindo-se a metodologia sugerida pela IOBC/WPRS (Hassan et al., 2000; Hassan \& Abdelgader, 2001).

O material biológico utilizado nos bioensaios foi constituído por parasitóides de ovos da espécie $T$. pretiosum, provenientes de criação mantida em laboratório, em câmaras climatizadas sob temperatura de $25 \pm 1{ }^{\circ} \mathrm{C}$, umidade relativa de $70 \pm 10 \%$ e fotofase de $14 \mathrm{~h}$, os 
quais foram multiplicados em ovos inviabilizados sob lâmpada germicida (Stein \& Parra, 1987) do hospedeiro Anagasta kuehniella-este criado conforme técnica descrita por Parra (1997).

Para cada bioensaio de toxicidade de herbicidas a $T$. pretiosum em seus estádios imaturos de desenvolvimento, foi utilizado um cartão contendo 60 círculos de $1 \mathrm{~cm}$ de diâmetro, com aproximadamente $350 \pm 50$ ovos por círculo, de no máximo $24 \mathrm{~h}$ de idade, do hospedeiro A. kuehniella, os quais foram expostos ao parasitismo por T. pretiosum. Após o parasitismo, os parasitóides foram descartados, e os cartões contendo ovos parasitados foram transferidos para cilindros de vidro e acondicionados em câmaras climatizadas nas mesmas condições da criação, até os parasitóides atingirem os períodos de desenvolvimento de $24 \mathrm{~h}$ ( $1 \mathrm{dia}$ ), $72 \mathrm{~h}$ (3 dias) e 168h (7 dias), correspondendo, respectivamente, aos estádios de ovo-larva, pré-pupa e pupa de $T$. pretiosum (Cônsoli et al., 1999).

Foram avaliados os herbicidas Zapp ${ }^{\circledR}$ Qi, Roundup $^{\circledR}$ WG, Roundup ${ }^{\circledR}$ (original) e Polaris ${ }^{\circledR}$ (bioensaio I); e Gliz ${ }^{\circledR} 480$ CS, Glifosato Nortox ${ }^{\circledR}$, Glifosato 480 Agripec $^{\circledR}$ e Roundup ${ }^{\circledR}$ Transorb (bioensaio II). Todas as formulações foram pulverizadas na concentração de $14,4 \mathrm{mg} \mathrm{L}^{-1}$ de equivalente ácido de glyphosate, correspondente à dose de $2,88 \mathrm{~kg} \mathrm{ha}^{-1}$ de glyphosate, e a testemunha foi constituída por água destilada.

As caldas herbicidas foram aplicadas sobre os círculos com os ovos do hospedeiro, contendo os parasitóides em diferentes estádios de desenvolvimento (ovo-larva, pré-pupa e pupa), com pulverizadores manuais de $580 \mathrm{~mL}$, que proporcionaram deposição de calda de $1,75 \pm 0,25 \mathrm{mg} \mathrm{cm}^{-2}$. A calibração foi realizada previamente à pulverização dos ovos, mediante pesagem do volume pulverizado em balança de precisão. Os ovos tratados permaneceram por cerca de três horas à temperatura ambiente, para evaporar o excesso de umidade produzido pela calda. Após esse período, os círculos com ovos foram transferidos para recipientes de vidro $(10 \mathrm{~cm}$ de comprimento e $2,5 \mathrm{~cm}$ de diâmetro) vedados na parte superior com tecido, preso com elástico, o que permitiu a ventilação e evitou a fuga dos parasitóides após a emergência. A porcentagem de emergência foi avaliada mediante contagem do número de adultos do parasitóide contido no recipiente de vidro, em relação ao número total de ovos parasitados contido no círculo de $1 \mathrm{~cm}$ de diâmetro. Ovos parasitados por Trichogramma adquirem coloração escura, quase preta, quando comparado a ovos não parasitados, característica que permite a identificação da ocorrência do parasitismo (Cônsoli et al., 1999).

Para os bioensaios de toxicidade de herbicidas sobre os adultos de $T$. pretiosum oriundos de ovos pulverizados, foram usadas gaiolas de exposição, que foram montadas utilizando-se duas placas de vidro, servindo como fundo e cobertura, e uma moldura de alumínio composta por quatro laterais $(13 \mathrm{~cm}$ de comprimento $\times 1,5 \mathrm{~cm}$ de altura $\times 1 \mathrm{~cm}$ de largura), que foram fixadas através de presilhas, conforme Hassan \& Abdelgader (2001). Em três laterais da moldura de aluminio havia seis orifícios para ventilação (diâmetro aproximado de $1 \mathrm{~cm}$ ). No quarto lado havia dois orificios: o maior $(3,5 \times 1 \mathrm{~cm})$ foi utilizado para introdução de ovos do hospedeiro A. kuehniella a serem parasitados e alimento ( $3 \mathrm{~g}$ de gelatina, $100 \mathrm{~mL}$ de água e $200 \mathrm{~g}$ de mel) aos insetos em teste; e o menor (diâmetro aproximado de $1 \mathrm{~cm}$ ) serviu para inserção dos tubos de emergência com os individuos a serem testados.

Tubos de vidro contendo adultos de T. pretiosum com cerca de 24 horas de idade foram conectados às gaiolas de exposição, permitindo a entrada dos insetos no interior destas. Para acelerar a saída dos insetos dos tubos de emergência após a conexão, a intensidade luminosa da sala foi aumentada de 500 para 2.500 lux por um período de 16 horas. Após esse periodo, os tubos de emergência foram desconectados e mantidos em condições controladas (temperatura de $25 \pm 1{ }^{\circ} \mathrm{C}$, umidade relativa de $70 \pm 10 \%$ e fotofase de $14 \mathrm{~h}$ ) por mais três dias, para que ocorresse a total emergência dos adultos, sendo estes utilizados no cálculo do número de indivíduos que entraram na gaiola. Um sistema de sucção de ar, constituído por bombas de aquário com fluxo invertido e mangueiras, foi conectado às gaiolas de exposição durante todo o período de condução do bioensaio, evitando o aumento na concentração de gases tóxicos no ambiente interno das gaiolas.

Seis horas após a desconexão dos tubos de emergência, cartões contendo três círculos de

Planta Daninha, Viçosa-MG, v. 26, n. 3, p. 611-617, 2008 
$1 \mathrm{~cm}$ de diâmetro com $400 \pm 50$ ovos inviabilizados de $A$. kuehniella e alimento foram oferecidos $96 \mathrm{~h}$ (três cartões), $120 \mathrm{~h}$ (dois cartões) e 168h (um cartão) após pulverização dos herbicidas, para serem parasitados pelas fêmeas de $T$. pretiosum e para avaliação da capacidade de parasitismo, totalizando um período de cerca de $150 \mathrm{~h}$ (aproximadamente seis dias) em que ovos do hospedeiro ficaram disponiveis para o parasitóide.

Sete dias após o início do bioensaio, as gaiolas foram desmontadas e os cartões contendo ovos do hospedeiro foram retirados e transferidos para placas de Petri descartáveis, sendo incubados nas mesmas condições do teste por mais três dias. Isso permitiu que todos os ovos parasitados se tornassem escuros, para posteriormente ser realizada a contagem.

$\mathrm{O}$ número de fêmeas em cada gaiola foi determinado por meio da contagem do número de ovos parasitados de cada círculo de $1 \mathrm{~cm}$ de diâmetro, utilizado na confecção do tubo de emergência, e multiplicado pelo número de parasitóides por ovo da população do inseto. $\mathrm{O}$ valor obtido foi subtraído do número de adultos que permaneceram no interior do tubo de emergência e multiplicado pela razão sexual da população. Os parâmetros número de parasitóides por ovo e razão sexual foram obtidos mediante avaliação de quatro círculos de $1 \mathrm{~cm}$ de diâmetro contendo ovos parasitados, sendo estes círculos retirados do mesmo cartão usado na confecção dos tubos de emergência do tratamento testemunha.

Com o número de ovos parasitados dividido pelo número de fêmeas que entraram na gaiola, calculou-se o número médio de ovos parasitados por fêmea de $T$. pretiosum de cada tratamento, que foi utilizado para mensurar a capacidade de parasitismo.

A análise estatística foi realizada da seguinte forma: nos bioensaios I e II, cada tratamento foi repetido quatro vezes para cada estádio (ovo-larva, pré-pupa e pupa); cada círculo com $350 \pm 50$ ovos parasitados foi considerado uma repetição no delineamento inteiramente casualizado, em esquema fatorial (cinco tratamentos $\mathrm{x}$ três estádios do parasitóide). Com base nas porcentagens de reduções de emergência (estádios imaturos) e reduções no parasitismo (adultos), os herbicidas testados foram classificados, segundo IOBC/WPRS, em: inócuo $(<30 \%)$; levemente nocivo $(30-79 \%)$; moderadamente nocivo (80-99\%); e nocivo (>99\%). A classificação dos herbicidas foi realizada em função do produto comercial, conforme Hassan et al. (2000). Os resultados obtidos foram submetidos à análise da variação e comparação de médias pelo teste de Tukey, ambos a $5 \%$ de probabilidade de erro.

\section{RESULTADOS E DISCUSSÃO}

Nos bioensaios com estádios imaturos (ovo-larva, pré-pupa e pupa) de T. pretiosum, somente foram verificadas diferenças para a variável porcentagem de emergência dos adultos de $T$. pretiosum no esdádio de ovo-larva do bioensaio I (Tabela 1). Houve efeito na emergência de adultos conforme o estádio de desenvolvimento para o herbicida Glifosato Agripec ${ }^{\circledR}$ quando pulverizado sobre o estádio pupal; diferindo da emergência, quando ele foi pulverizado sobre o estádio de pré-pupa no bioensaio II (Tabela 1).

Na Tabela 2 são apresentadas as reduções na emergência (RE) de adultos de $T$. pretiosum, para os respectivos estádios de desenvolvimento avaliados, e a aplicação da classificação proposta pela IOBC/WPRS para testes de toxicidade com parasitóides. Dos oito herbicidas avaliados, mesmo apresentando reduções distintas entre os tratamentos e estádios de desenvolvimento, todos foram classificados como classe 1 (inócuo), ou seja, não causaram reduções acima de 30\% na emergência de adultos do parasitóide, apresentando redução entre 0,00 e 23,18\% (Tabela 2, bioensaios I e II).

No bioensaio I, no estádio de ovo-larva, todos os tratamentos reduziram a emergência; os produtos Zapp ${ }^{\circledR}$ Qi e Polaris ${ }^{\circledR}$ apresentaram maior efeito, reduzindo 13,68 e $23,18 \%$, respectivamente (Tabela 2). Já os produtos Roundup $\mathrm{WG}^{\circledR}$ e Roundup ${ }^{\circledR}$ (original) apresentaram maior RE no estádio de pré-pupa, quando comparado ao de ovo-larva. No bioensaio II, apenas o produto Roundup Transorb ${ }^{\circledR}$ apresentou RE nos três estádios de desenvolvimento do parasitóide, sendo o estádio de ovo-larva o mais sensivel, com RE de $12,03 \%$, enquanto para prépupa e pupa a RE foi de 4,66 e 1,19\%, respectivamente. O produto comercial Glifosato Nortox ${ }^{\mathbb{R}}$ foi o único a não reduzir a emergência em 
Tabela 1 - Emergência (\%) de adultos de Trichogramma pretiosum em ovos do hospedeiro Anagasta kuehniella, contendo o parasitóide em diferentes estádios de desenvolvimento. Temperatura: $25 \pm 1{ }^{\circ} \mathrm{C}$; UR: $70 \pm 10 \%$; fotofase: 14h; luminosidade: 500 lux. FAEM/DFs/UFPel, Capão do Leão-RS, 2005/2006

\begin{tabular}{|c|c|c|c|c|}
\hline \multirow{2}{*}{ Tratamento } & \multirow{2}{*}{$\mathrm{DC}^{1 /}$} & \multicolumn{3}{|c|}{ Estádio de desenvolvimento ${ }^{\underline{2}}$} \\
\hline & & Ovo-larva & Pré-pupa & Pupa \\
\hline \multicolumn{5}{|l|}{ Bioensaio I } \\
\hline Testemunha & - & $115,20 \mathrm{a} \mathrm{A}$ & 112,02 a A & 98,62 a A \\
\hline Zapp $^{\circledR} \mathrm{Qi}$ & 5,8 & $99,44 \mathrm{ab} \mathrm{A}$ & 114,08 a A & 102,55 a A \\
\hline Roundup $^{\mathbb{R}}$ WG & 4 & $111,09 \mathrm{ab} \mathrm{A}$ & $98,73 \mathrm{a} \mathrm{A}$ & 95,06 a A \\
\hline Roundup $^{\circledR}$ (original) & 8 & $108,15 \mathrm{ab} A$ & 101,17 a A & 98,84 a A \\
\hline Polaris $^{(\mathbb{R}}$ & 8 & $88,50 \mathrm{~b} \mathrm{~A}$ & 93,41 a A & 97,93 a A \\
\hline \multicolumn{5}{|l|}{ Bioensaio II } \\
\hline Testemunha & - & 108,62 a A & 119,55 a $\mathrm{A}$ & 112,13 a A \\
\hline $\mathrm{Gliz}^{\mathbb{B}} 480 \mathrm{CS}$ & 6 & $108,11 \mathrm{a} \mathrm{A}$ & $129,93 \mathrm{a} \mathrm{A}$ & 129,39 a A \\
\hline Glifosato Nortox $^{\circledR}$ & 6 & $111,88 \mathrm{a} \mathrm{A}$ & 130,38 a A & 126,45 a A \\
\hline Glifosato 480 A gripec $^{(\mathbb{B}}$ & 6 & $109,58 \mathrm{a} A B$ & 32,72 a A & 105,63 a B \\
\hline Roundup ${ }^{\mathbb{B}}$ Transorb & 6 & $95,55 \mathrm{a} \mathrm{A}$ & 113,97 a A & 110,79 a A \\
\hline
\end{tabular}

${ }_{1 /}$ Dose do produto comercial ( $\mathrm{kg}$ ou $\mathrm{L} \mathrm{ha}^{-1}$ ). Todas as formulações foram pulverizadas na concentração de $14,4 \mathrm{mg} \mathrm{L}^{-1}$ de equivalente ácido de glyphosate, correspondente à dose de $2,88 \mathrm{~kg} \mathrm{ha}^{-1} \mathrm{de}$ glyphosate. ${ }^{2 /}$ Tempo após parasitismo: ovo-larva $(24 \mathrm{~h})$, prépupa $(72 \mathrm{~h})$ e pupa $(168 \mathrm{~h})$. Médias acompanhadas por letras idênticas, minúsculas na coluna e maiúsculas na linha, não diferem significativamente $(\mathrm{p}>0,05)$ pelo teste de Tukey a $5 \%$ de probabilidade.

todos os estádios de T. pretiosum. Esses resultados propiciam indícios de que a diferenciação entre os ingredientes inertes e o tipo de sal presente na formulação do glyphosate pode constituir um fator preponderante para a toxicidade sobre o parasitóide $T$. pretiosum.

Comparando os resultados obtidos entre os estádios de desenvolvimento de ovo-larva, prépupa e pupa dos bioensaios I e II (Tabela 2), observa-se que a porcentagem de redução na emergência de adultos de $T$. pretiosum é menor no estádio de pupa, com exceção do produto Glifosato 480 Agripec $^{\circledR}$, que mostrou redução maior neste estádio, enquanto nos outros dois estádios não houve redução. Resultados para emergência de adultos de $T$. pretiosum foram previamente relatados para Glifosato Nortox $^{\circledR}$ por Giolo et al. (2007), os quais observaram uma porcentagem de emergência de 102,20, 83,00 e $87,7 \%$ para as fases de ovo-larva, prépupa e pupa, respectivamente, sendo inferiores aos do presente trabalho, que foram de $111,88,130,38$ e $126,45 \%$ (Tabela 1, bioensaio II), indicando a emergência de mais de
Tabela 2 - Redução na emergência (RE\%) de adultos de Trichogramma pretiosum em ovos do hospedeiro Anagasta kuehniella, contendo o parasitóide em diferentes estádios de desenvolvimento e classificação de toxicidade. Temperatura: $25 \pm 1{ }^{\circ} \mathrm{C}$; UR: $70 \pm 10 \%$; fotofase: $14 \mathrm{~h}$; luminosidade: 500 lux. FAEM/DFs/UFPel, Capão do LeãoRS, 2005/2006

\begin{tabular}{|c|c|c|c|c|c|c|}
\hline \multirow{3}{*}{ Tratamento } & \multicolumn{6}{|c|}{ Estádio de desenvolvimento } \\
\hline & \multicolumn{2}{|c|}{ Ovo-larva } & \multicolumn{2}{|c|}{ Pré-pupa } & \multicolumn{2}{|c|}{ Pupa } \\
\hline & $\mathrm{RE}^{1 / /}$ & Classe $^{2}$ & $\mathrm{RE}^{1 /}$ & Classe & $\mathrm{RE}^{1 /}$ & Classe $^{2}$ \\
\hline \multicolumn{7}{|l|}{ Bioensaio I } \\
\hline Zapp $^{\sqrt{8}} \mathrm{Qi}^{\mathrm{N}}$ & 13,68 & 1 & 0 & 1 & 0 & 1 \\
\hline Roundup $^{\sqrt{8}} \mathrm{WG}$ & 3,57 & 1 & 11,87 & 1 & 6,61 & 1 \\
\hline Roundup $^{\mathbb{R}}$ (original) & 6,12 & 1 & 9,69 & 1 & 0 & 1 \\
\hline Polaris $^{\mathbb{R}}$ & 23,18 & 1 & 16,62 & 1 & 0,7 & 1 \\
\hline \multicolumn{7}{|l|}{ Bioensaio II } \\
\hline Gliz $480 \mathrm{CS}$ & 0,47 & 1 & 0 & 1 & 0 & 1 \\
\hline Glifosato Nortox & 0 & 1 & 0 & 1 & 0 & 1 \\
\hline Glifosato 480 Agripec & 0 & 1 & 0 & 1 & 5,8 & 1 \\
\hline Roundup $^{\text {BR }}$ Transorb & 12,03 & 1 & 4,66 & 1 & 1,19 & 1 \\
\hline
\end{tabular}

1/ Redução na emergência de adultos comparada com a testemunha do bioensaio. ${ }^{2 /}$ classes da IOBC/WPRS para teste de toxicidade sobre Trichogramma pretiosum em seus estádios imaturos: 1, inócuo $(<30 \%) ; 2$, levemente nocivo $(30-79 \%) ; 3$, moderadamente nocivo (80-99\%); e 4 , nocivo ( $>99 \%)$.

um parasitóide por ovo (> 100\%). Resultados semelhantes aos do presente trabalho foram observados por Manzoni et al. (2007), que avaliaram quatro formulações de glyphosate $\left(\right.$ Gliz $^{\circledR} 480$ CS, Glifosato Nortox ${ }^{\circledR}$, Roundup ${ }^{\circledR}$ (original) e Roundup $\mathrm{WG}^{\circledR}$ ), sendo todos considerados inócuos (classe 1).

Quando se avaliou a toxicidade dos herbicidas sobre os adultos de $T$. pretiosum oriundos de pupas pulverizadas, os resultados dos bioensaios não apresentaram diferenças significativas quanto ao parasitismo entre os tratamentos (Tabela 3). Os herbicidas que causaram reduções no parasitismo de $T$. pretiosum variaram de 2,50 a $16,38 \%$, sendo todos classificados como inócuos (classe 1) (Tabela 3, bioensaios I e II).

Resultados similares para Roundup ${ }^{\circledR}$ (original) sobre adultos foram obtidos por Manzoni et al. (2006), embora tenham utilizado $12 \mathrm{~L} \mathrm{ha}^{-1}$ do produto comercial. O herbicida Roundup ${ }^{\circledR}$ WG (1,26\% glyphosate) também foi classificado como moderadamente nocivo (classe 3) a adultos de $T$. pretiosum por Manzoni et al. (2007).

Planta Daninha, Viçosa-MG, v. 26, n. 3, p. 611-617, 2008 
Tabela 3 - Número de ovos parasitados ( \pm EP) por fêmea, redução no parasitismo (\%) de adultos de Trichogramma pretiosum e classificação de toxicidade. Temperatura $25 \pm 1{ }^{\circ} \mathrm{C}$; UR: $70 \pm 10 \%$; fotofase: $14 \mathrm{~h}$; luminosidade 500 lux. Pelotas-RS. 2006/2007

\begin{tabular}{|c|c|c|c|}
\hline Tratamento & Ovos por fêmea ${ }^{\underline{1} /}$ & $\mathrm{RP}^{2 / /}$ & Classe $\mathrm{IOBC}^{3 /}$ \\
\hline \multicolumn{4}{|l|}{ Bioensaio I } \\
\hline Testemunha & $42,43 \mathrm{a}$ & - & - \\
\hline $\operatorname{Zapp}^{(B)} \mathrm{Qi}$ & $42,46 \mathrm{a}$ & 0 & 1 \\
\hline Roundup ${ }^{\circledR}$ WG & $43,37 \mathrm{a}$ & 0 & 1 \\
\hline Roundup $^{\circledR}$ (original) & $40,07 \mathrm{a}$ & 5,56 & 1 \\
\hline Polaris $^{(\mathbb{R}}$ & $36,78 \mathrm{a}$ & 13,31 & 1 \\
\hline \multicolumn{4}{|l|}{ Bioensaio I } \\
\hline Testemunha & $33,02 \mathrm{a}$ & - & - \\
\hline $\mathrm{Gliz}^{(B)} 480 \mathrm{CS}$ & $32,20 \mathrm{a}$ & 2,50 & 1 \\
\hline Glifosato Nortox $^{\circledR}$ & $27,61 \mathrm{a}$ & 16,38 & 1 \\
\hline Glifosato 480 Agripec $^{\circledR}$ & $39,66 \mathrm{a}$ & 0 & 1 \\
\hline Roundup $^{\circledR}$ Transorb & $33,97 \mathrm{a}$ & 0 & 1 \\
\hline
\end{tabular}

1/ Médias acompanhadas por letras idênticas não diferem significativamente $(p>0,05)$ pelo teste de Tukey a 5\% de probabilidade; ${ }^{2} /$ redução no parasitismo, comparado com a testemunha negativa (água destilada) do experimento; ${ }^{3}$ classes da IOBC/WPRS para teste de toxicidade sobre Trichogramma pretiosum em adultos: classe 1 , inócuo $(<30 \%) ; 2$, levemente nocivo (30-79\%); 3 , moderadamente nocivo $(80-99 \%)$; e 4 , nocivo $(>99 \%)$

Resultados distintos foram observados por Hassan et al. (1988), ao classificarem o herbicida Roundup ${ }^{\circledR}(0,360 \%$ de glyphosate) como levemente nocivo aos adultos de $T$. cacoeciae. Giolo et al. (2005) classificaram Roundup ${ }^{\circledR}$ WG como levemente nocivo (classe 2) a adultos de $T$. pretiosum ao obterem redução no parasitismo de $61,56 \%$, superior à encontrada no presente trabalho, e os produtos Polaris ${ }^{\circledR}$ e Roundup Transorb ${ }^{\circledR}$ foram classificados como moderadamente nocivos (classe 3 ) a adultos de $T$. pretiosum, na mesma concentração de $14,4 \mathrm{mg} \mathrm{L}^{-1}$ de equivalente ácido de glyphosate utilizada neste trabalho. Da mesma forma, também foram observados efeitos deletérios de Glifosato Nortox $^{\circledR}(1,08 \%$ de glyphosate $)$ e Gliz $^{\circledR} 480$ CS (1,08\% de glyphosate) sobre T. pretiosum por Manzoni et al. (2007), porém com toxicidade superior (classe 3) à encontrada no presente trabalho (classe 1) para ambos os herbicidas (Tabela 3).

As diferenças nos resultados obtidos neste trabalho podem estar relacionadas a características intrínsecas do ovo, visto que neste experimento a avaliação da redução no parasitismo foi realizada com parasitóides adultos emergentes provenientes de ovos pulverizados no estádio de pupa do parasitóide. Verificouse que os parasitóides adultos avaliados sofreram ação dos tratamentos na fase imatura, de maneira distinta dos trabalhos citados, em que eles foram expostos diretamente aos resíduos das formulações comerciais dos herbicidas. Pode-se destacar que os resultados encontrados neste trabalho com adultos emergentes vão de encontro aos resultados obtidos com as fases de ovo-larva, pré-pupa e pupa, em que todos os herbicidas foram classificados como inócuo (classe 1) (Tabela 3, bioensaios I e II).

Com relação aos ovos do hospedeiro, características como permeabilidade do córion e das camadas adjacentes são variáveis de acordo com a espécie, a idade e o estádio de desenvolvimento do ovo (Cônsoli et al., 1999). Nesse sentido, esses autores estudaram as estruturas de ovos de seis espécies de lepidópteros, incluindo A. kuehniella, e verificaram diferenças intra-específicas na espessura do córion, especialmente do exocórion, e na densidade protéica desta última camada, a qual, provavelmente, foi um dos fatores responsáveis pela não-penetração dos tratamentos com herbicidas.

Com base nos resultados apresentados neste estudo, pode-se concluir que os herbicidas comerciais com formulações à base de glyphosate são seletivos aos estádios imaturos do parasitóide de ovos $T$. pretiosum, além de não afetarem a capacidade de parasitismo de adultos emergidos de ovos de A. kuehniella quando o parasitóide se encontra na fase de pupa. Além do mais, ainda se pode relacionar tais resultados com aqueles obtidos a partir das mesmas formulações comerciais testadas por Giolo et al. (2005), sobre adultos, sendo possivel, dessa forma, utilizar esses produtos no manejo de pragas de culturas anuais e frutíferas.

\section{AGRADECIMENTOS}

Ao Conselho Nacional de Desenvolvimento Científico e Tecnológico do Brasil (CNPq-Brasil) e à Fundação de Amparo à Pesquisa do Estado do Rio Grande do Sul (FAPERGS), pelo apoio financeiro e pelas concessões de bolsas. 


\section{LITERATURA CITADA}

AMARANTE JÚNIOR, O. P.; SANTOS, T. C. R Glifosato: propriedades, toxicidade, usos e legislação. Química Nova, v. 25, n. 4, p. 589-593, 2002.

BOTELHO, P. M. Eficiência de Trichogramma em campo. In: PARRA, J. R. P.; ZUCCHI, R. A. (Eds.). Trichogramma e o controle biológico aplicado. Piracicaba: FEALQ, 1997. p. 303-318.

CARVALHO, G. A.; PARRA, J. R. P.; BAPTISTA, G. C. Seletividade de alguns produtos fitossanitários a duas linhagens de Trichogramma pretiosum Riley, 1879 (Hymenoptera: Trichogrammatidae). Ci. Agrotec., v. 25, n. 3 , p. $583-591,2001$.

CÔNSOLI, F. L.; ROSSI, M. M.; PARRA, J. R. P. Developmental time and characteristics of the immature stages of Trichogramma galloi and Trichogramma pretiosum Riley (Hymenoptera, Trichogrammatidae). R. Bras.

Entomol., v. 43, n. 2, p. 271-275, 1999.

GIOLO, F. P. et al. Seletividade de formulações de glyphosate a Trichogramma pretiosum (Hymenoptera: Trichogrammatidae). Planta Daninha, v. 23, n. 3, p. 457462, 2005.

GIOLO, F. P. et al. Toxicidade de pesticidas utilizados na cultura do pessegueiro para estádios imaturos de Trichogramma pretiosum Riley (Hymenoptera: Trichogrammatidae). BioAssay, v. 1, n. 4, p. 1-7, 2006. Disponível em: <http://www.bioassay.org.br/articles/1.4/ BA1.4.pdf>. Acesso em: 6 de jun. de 2007.

HASSAN, S. A. Guidelines for the evaluation of side effects of plant protection products on Trichogramma cacoeciae Marchal (Hym., Trichogrammatidae). IOBC/WPRS B. v. 21, n. 6, p. 119-128, 1998.

HASSAN, S. A.; ABDELGADER, H. A sequential testing program to assess the effects of pesticides on Trichogramma cacoeciae Marchal (Hym. Trichogrammatidae). Pesticides and Beneficial Organisms. IOBC/WPRS B., v. 24, n. 4, p. 71-81, 2001.

HASSAN, S. A. et al. Results of the fourth joint pesticide testing programme carried out by the IOBC/WPRS Working Group "Pesticides and Beneficial Organisms". Z. Ang. Entomol., v. 105, p. 321-329, 1988.

HASSAN, S. A. et al. The side-effects of pesticides on the egg parasitoid Trichogramma cacoeciae Marchal (Hym., Trichogrammatidae), acute dose-response and persistence tests. J. Appl. Entomol., v. 122, p. 569-573, 1998.
HASSAN, S. A. et al. A laboratory method to evaluate the side effects of plant protection products on Trichogramma cacoeciae Marchal (Hym., Trichogrammatidae). In: CANDOLFI, M. P. et al. (Eds.). Guidelines to evaluate side-effects of plant protection products to non-target arthropods. Reinheim: IOBC/WPRS, 2000. p. 107-119.

MANZONI, C. G. et al. Seletividade de agrotóxicos usados na produção integrada de maçã para adultos de Trichogramma pretiosum. Pesq. Agropec. Bras., v. 41, n. 10, p. $1461-1467,2006$.

MANZONI, C. G. et al. Seletividade de agroquímicos utilizados na produção integrada de maçã aos parasitóides Trichogramma pretiosum Riley e Trichogramma atopovirilia Oatman \& Platner (Hymenoptera: Trichogrammatidae). Bioassay, v. 2, n. 1, p. 1-11, 2007. Disponível em: $<\mathrm{http}$ ://www. bioassay.org.br/articles/2.1/ BA2.1.pdf $>$ Acesso em: 3 de mar. de 2007.

PARRA, J. R. P. Técnicas de criação de Anagasta kuehniella, hospedeiro alternativo para produção de Trichogramma. In: PARRA, J. R. P.; ZUCCHI, R. A. (Eds.). Trichogramma e o controle biológico aplicado. Piracicaba: FEALQ, 1997. p. $121-150$.

RIZZARDI, M. A. et al. Ação de herbicidas sobre mecanismos de defesa das plantas aos patógenos. Ci. Rural, v. 33, n. 5, p. 957-965, 2003.

RODRIGUES, B. N.; ALMEIDA, F. S. Guia de herbicidas. 5.ed. Londrina: Grafmarke, 2005. 591 p.

SANTOS, J. B. et al. Tolerance of Bradyrhizobium strains to glyphosate formulations. Crop Protec., v. 24, n. 3, p. 543$547,2005$.

SANTOS, J. B. et al. Action of two herbicides on the microbial activity of soil cultivated with common bean (Phaseolus vulgaris) in conventional-till and no-till systems. Weed Res., v. 46, p. 1-6, 2006.

SHANER, D. L. The impact of glyphosate-tolerant crops on the use of other herbicides and on resistance management. Pest Manag. Sci., v. 56, n. 4, p. 320-326, 2000.

SIQUEIRA, J. O. et al. Interferências no agrossistema e riscos ambientais de culturas transgênicas tolerantes a herbicidas e protegidas contra insetos. Cader. Ci. Tecnol., v. 21 , p. $11-81,2004$

STEIN, C. P.; PARRA, J. R. P. Uso da radiação ultravioleta para inviabilizar ovos de Anagasta kuehniella (Zeller, 1879) visando estudos com Trichogramma spp. An. Soc.

Entomol. Brasil, v. 16, n. 1, p. 229-233, 1987.

ZUCCHI, R. A.; MONTEIRO, R. C. O gênero Trichogramma na América do Sul. In: PARRA, J. R. P.; ZUCCHI, R. A. (Eds.). Trichogramma e o controle biológico aplicado. Piracicaba: FEALQ, 1997. p. 41-66. 